\title{
From Outage Probability to ALOHA MAC Layer Performance Analysis in Distributed WSNs
}

\author{
Mohamed-El-Amine Seddik ${ }^{1,2}$, Viktor Toldov ${ }^{2,3}$, Laurent Clavier ${ }^{1,3}$, Nathalie Mitton ${ }^{2}$ \\ ${ }^{1}$ IMT Lille-Douai, ${ }^{2}$ Inria, ${ }^{3}$ IRCICA USR CNRS 3380 \\ mseddik@etu.imt-lille-douai.fr
}

\begin{abstract}
In cellular networks, the outage probability is the probability that the signal-to-interference-plus-noise-ratio (SINR) is less than a given threshold. In this article, firstly, we develop an explicit formula for outage probability for a wireless sensor network taking account of the problem parameters - i.e. the spatial density of nodes in the network, the noise power, the transmission power, the distance between the considered node and the base station, and finally the attenuation coefficient -. Secondly, we model a given node by a Markov chain to study the performance of the MAC layer in the case of a SlottedALOHA protocol and Slotted-ALOHA with preliminary channel reservation. Especially in this study, we theoretically estimate the transmission success likelihood after a certain number of attempts that we consider as a metric to evaluate the performance of the considered protocol. Finally we compare the theoretical results with experimental ones achieved on the large scale platform FIT IoT-Lab of Lille. To sum up, this paper responds to the following question: How many channels do we need to a achieve high performance distributed wireless sensor network? The answer is given by the necessary number of channels which maximizes the transmission success likelihood after a certain number of trials.
\end{abstract}

\section{INTRODUCTION}

Wireless sensor networks (WSN) are beginning to be deployed at an accelerated pace [1] and it is totally reasonable to believe that in few years the world will be covered with wireless sensor networks due to the unlimited potential of applications offered by this new technology. However, despite these advantages, there is a number of challenges to face before getting high performances in totally distributed WSN. Indeed, we can mention the problem of energy, which is mainly the most important to focus on, as a WSN could be deployed in inaccessible areas, where it is impossible to change the batteries of nodes [2]. It is thus essential to develop techniques that keep the network up for several years. There are multiple complementary approaches to tackle this technological challenge, like finding new sources of energy (solar for example), or reducing nodes consumption by optimizing the hardware and the software. Besides, the performance of WSN is influenced by the interference [3], [4] resulting in packet drops, re-transmissions and inconsistent protocol behavior. As a consequence, interference is partially responsible for the loss of energy. Interference is closely linked to both MAC and physical layers and in terms of hardware, interference is linked to the radio communication part, which is the unit that consumes most of energy [5]. Thus, it is essential to decrease its use by reducing for instance its active time. A way to achieve it is to avoid re-transmissions as much as possible by better handling interference and collision. This implies to review the development of the different communication layers and essentially the MAC layer, responsible for controlling the medium access and the radio duty cycling (RDC). Another important factor is the nodes spatial distribution, which increases interference in some configurations and therefore degrades performances [6]. In this work, we take this aspect into account by modeling the nodes as a collection of points distributed according to a Poisson Point Process [7]. In this paper, we focus on the software aspect, that is to say, through a theoretical study and experiments with real nodes, we estimate the performances of the Slotted-ALOHA MAC protocol with channel reservation part in terms of success in packets transmissions taking interference into account.

The contributions of this paper is three-fold:

- Considering a Slotted-ALOHA MAC protocol, we propose a formula for the outage probability for a given node of the network.

- Thanks to a Markovian approach, we define a metric to allow the performances evaluation of the Slotted-ALOHA protocol with channel reservation.

- We compare these theoretical results to practical ones using FIT IoT-Lab platform [8].

Thus, the purpose of this paper is to take advantage of the outage information to estimate the performance of the SlottedALOHA MAC protocol with channel reservation, in order to get a criterion about the number of necessary channels that we need and thus, to achieve a high performance distributed wireless sensor network. In addition, our findings have been experimentally verified. To the best of our knowledge, this is the first study that provides such results.

The remainder of this paper is organized as follows. First, we present the context in Section II and second discuss the related work in Section III. In Section IV, we develop a formula of outage probability related to the considered context. Then, in Section V, by considering a Slotted-ALOHA protocol with channel reservation we develop a model based on Markov chain to study the performances of the considered protocol in terms of transmission success. In Section VI, we consider the case when interfering nodes are present in the range's outer region of the base station. At the end, in order to validate this model, we compare the obtained results with practical ones achieved in the large scale FIT-IoT-Lab platform of Lille [8]. 


\section{Problem Statement}

Let us consider a wireless sensor network composed of a certain number of nodes and a unique base station (BTS) as shown in Fig. 1. On this figure, the BTS is represented by a blue star characterized by the blue circle which represents its range area. We consider a communicating node (green square) at distance $r$ from the BTS and assume that it is in the BTS's range as illustrated. Finally the red dots represent interfering nodes. We suppose that the role of this BTS is restricted to collect incoming packets. We consider a distributed access policy where the BTS do not transmit any signaling packets to the nodes that make the decision to transmit on their own. Nodes should share a given number of channels and each of them has to choose one channel randomly and send its packets on it. Thus, it might be possible, that two or more nodes choose the same channel. In this case, the communication between a given node and the BTS may be interrupted and the transmitted packets may be lost because of collisions.

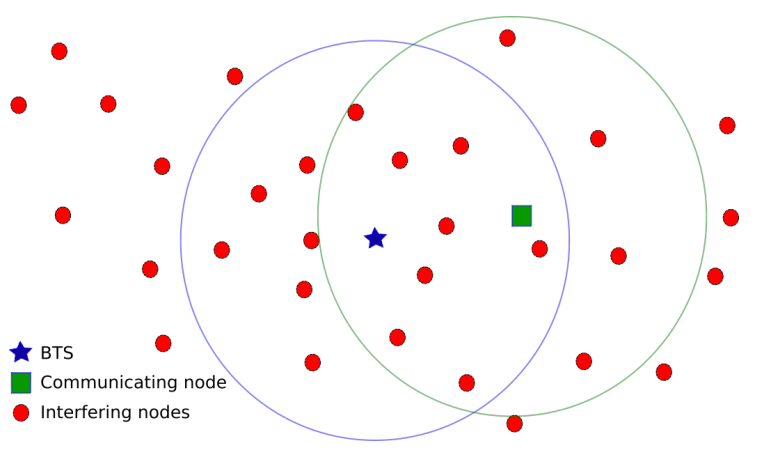

Fig. 1. Illustration of a distributed network when considering a scenario of interference.

\section{RELATED WORKS}

To model the problem of interference, the notion of outage probability has been introduced. There has been a significant number of work in the literature around the estimation of the outage probability. In particular, in [9], [10]. S. Weber analyzes the phenomenon of interference in ad-hoc networks in terms of transmission capacity which derives from the outage probability. In [11], Z. Gong gives, based on the estimation of outage probability in mobile random networks, results that show the importance of routing, MAC and retransmissions schemes to avoid bursts of transmission failures. [12] presents a comprehensive survey on the literature related to stochastic geometry models which are widely used to model large-scale ad-hoc wireless networks, in particular, the outage probability is derived generally from these models. By means of this literature, the calculation of a closed form formula of outage probability is still intractable since it requires knowing the exact probability density function (PDF) of the interference signal. Besides, it is possible to model the interference signal as an alpha-stable model [10], in which case, one has the exact formula of the characteristic function $(\mathrm{CF})$ and thus one has access to the PDF as the Fourier transform of the CF. However, in general a closed form expression for the corresponding PDF can not be obtained and thus one has to use numerical methods. In this paper, rather than estimating the PDF of the interference signal, we propose a sampling-based approach by expressing the interference signal in terms of uniform random variables and thus consistently estimate the outage probability.

We then use the outage information to estimate the performance of the Slotted-ALOHA MAC protocol [13] with channel reservation. Thanks to a Markovian approach, we define a metric to allow the performance evaluation which is the transmission success likelihood after a certain allowed number of attempts, of a new node trying to join the network.

\section{Outage Probability}

\section{A. Definition of $O p$}

In multiple-access distributed networks, interference could be a huge issue that interrupts communications between different nodes. To model the problem of interference, the notion of outage probability has been introduced. According to [14] the outage probability is defined as the probability that the signal-to-interference-plus-noise-ratio (SINR) is less than a given threshold $\tau$, formally we denote $O p$ as:

$$
O p=\mathbb{P}(S I N R<\tau)
$$

\section{B. Assumptions}

Assumption 1. We assume that the nodes distribution follows a Homogeneous Poisson Point Process (HPPP) with spatial density $\lambda$, which we denote by $\Pi_{d, \lambda}$ and where $d=2$ is the dimension of the points.

Assumption 2. We assume that the wireless channel between the BTS and any node $X_{i} \in \Pi_{d, \lambda}$ consists of path loss attenuation with no fading. Formally $P_{r, i}=P_{e}\left\|X_{i}\right\|^{-\alpha}$, where $\alpha$ is the attenuation coefficient, $P_{r, i}$ is the received power by the BTS from the node $X_{i}$ and $P_{e}$ holds for the emission power, which is assumed to be the same for all the nodes in the network.

Assumption 3. We assume that the Medium Access Control strategy is a Slotted-ALOHA: All nodes are synchronized on the same temporal base and each node $X_{i} \in \Pi_{2, \lambda}$ sends a packet of duration $T_{s}$ after each time interval $T$. Using a number $N_{c}$ of frequencies, during this interval the communicating nodes have a density $\lambda^{*}$ such as:

$$
\lambda^{*}=\frac{2 T_{s}}{T} \frac{1}{N_{c}} \lambda
$$

\section{Expression of $O p$}

We consider a direct orthonormal reference linked to the plan $\mathbb{R}^{2}$. Without loss of generality, we assume that the BTS is located at the origin $(o)$ of this reference. We consider a node located at a distance $r$ from the origin, the $S I N R$ is given by the following formula:

$$
S I N R(o, r)=S(o, r) /\left(I(o)+N_{0}\right)
$$


where $S(o, r)$ represents the useful signal power received by the BTS of the signal transmitted by the node at distance $r$ from the BTS, $I(o)$ denotes the interference signal at point (o) generated by the interfering nodes and $N_{0}$ represents the noise power. As assumed in Assumption 2, the wireless channel between the BTS and any node consists of path loss attenuation with no fading, more formally:

$$
S(o, r)=P_{e} r^{-\alpha}, I(o)=\sum_{X_{i} \in \Pi_{\lambda}} P_{e}\left\|X_{i}\right\|^{-\alpha}
$$

The SINR is then expressed as follows:

$$
\operatorname{SINR}(o, r)=\frac{P_{e} r^{-\alpha}}{P_{e} \sum_{X_{i} \in \Pi_{\lambda}}\left\|X_{i}\right\|^{-\alpha}+N_{0}}
$$

Combining Equations (1) and (4), $O p$ can be expressed as:

$$
O p=\mathbb{P}\left(\sum_{X_{i} \in \Pi_{\lambda}}\left\|X_{i}\right\|^{-\alpha}>\frac{r^{-\alpha}}{\tau}-\frac{N_{0}}{P_{e}}\right)
$$

Looking to (5) the term $\sum_{X_{i} \in \Pi_{\lambda}}\left\|X_{i}\right\|^{-\alpha}$ is the only random variable (r.v.) since the collection of points is distributed as a HPPP according to Assumption 1. We denote this r.v. by $S_{\alpha, \lambda}$ and introduce $\xi=\frac{r^{-\alpha}}{\tau}-\frac{N_{0}}{P_{e}}$ then:

$$
O p=\mathbb{P}\left(S_{\alpha, \lambda}>\xi\right)
$$

At this stage, we do need to express the r.v. $S_{\alpha, \lambda}$. We prove in following a couple of lemmas that are essential.

Lemma IV.1. Let $\left(X_{k}\right)_{k \in \mathbb{N}} \in \Pi_{\lambda}$ be a HPPP with intensity $\lambda$ such that $\forall k \in \mathbb{N},\left\|X_{k}\right\|<\left\|X_{k+1}\right\|$. Then:

$$
\forall k \in \mathbb{N}, \mathbb{P}\left(\left\|X_{k}\right\|>r\right)=\frac{\left(\lambda \pi r^{2}\right)^{k}}{k !} \exp \left(-\lambda \pi r^{2}\right)
$$

Proof. $\left(X_{k}\right)_{k \in \mathbb{N}}$ being a HPPP with intensity $\lambda$, the number of points in a subset $A$ of $\mathbb{R}^{2}$ is a Poisson process with mean $\lambda|A|$, formally we have:

$$
\forall A \subset \mathbb{R}^{2}, \Pi_{\lambda}(A) \sim \operatorname{Poiss}(\lambda|A|)
$$

Let $B(o, r)$ be the ball of center $o$ and radius $r, \forall k \in \mathbb{N}$ we have:

$$
P\left(\Pi_{\lambda}(B(o, r))=k\right)=P\left(\left\|X_{k}\right\|>r\right)
$$

Besides:

$$
P\left(\Pi_{\lambda}(B(o, r))=k\right)=\frac{(\lambda|B(o, r)|)^{k}}{k !} \exp (-\lambda|B(o, r)|)
$$

and $|B(o, r)|=\pi r^{2}$ yields formula (7).

Lemma IV.2. Let $k \in \mathbb{N}$, the random variable (r.v.) $\left\|X_{k}\right\|$ can be generated in function of a uniform distributed r.v. $U_{k} \sim$ $U([0,1])$. We have the following expressions:

$$
\begin{gathered}
\left\|X_{0}\right\|=\sqrt{\frac{1}{\lambda \pi} \log \left(\frac{1}{1-U_{0}}\right)} \\
\forall k \geqslant 1,\left\|X_{k}\right\|=\sqrt{\frac{-k}{\lambda \pi} W_{0}\left[-\frac{1}{k} \exp \left(\frac{\log \left[\left(1-U_{k}\right) k !\right]}{k}\right)\right]}
\end{gathered}
$$

Where $W_{0}$ is the Lambert function defined such that:

$$
\forall x \in \mathbb{R}, W_{0}(x) e^{W_{0}(x)}=x
$$

Proof. Let $k \in \mathbb{N}$. Using Lemma IV.1, the cumulative distribution function of $\left\|X_{k}\right\|$ is given by the following expression:

$$
F_{\left\|X_{k}\right\|}(x)=1-\mathbb{P}\left(\left\|X_{k}\right\|>x\right)=1-\frac{\left(\lambda \pi x^{2}\right)^{k}}{k !} \exp \left(-\lambda \pi x^{2}\right)
$$

Let $U_{k} \sim U([0,1])$, using the inverse of the cumulative distribution function below, we can generate $\left\|X_{k}\right\|$ in function of $U_{k}$ as

$$
\left\|X_{k}\right\|=F_{\left\|X_{k}\right\|}^{-1}\left(U_{k}\right)
$$

In the case where $k=0$ :

$$
\left\|X_{0}\right\|=F_{\left\|X_{0}\right\|}^{-1}\left(U_{0}\right)=\sqrt{\frac{1}{\lambda \pi} \log \left(\frac{1}{1-U_{0}}\right)}
$$

Otherwise:

$$
\begin{gathered}
y=F_{\left\|X_{k}\right\|}(x)=1-\frac{\left(\lambda \pi x^{2}\right)^{k}}{k !} \exp \left(-\lambda \pi x^{2}\right) \\
\Rightarrow-\frac{\lambda \pi x^{2}}{k}=W_{0}\left[-\frac{1}{k} \exp \left(\frac{\log [(1-y) k !]}{k}\right)\right]
\end{gathered}
$$

Finally we get:

$$
F_{\left\|X_{k}\right\|}^{-1}(x)=\sqrt{\frac{-k}{\lambda \pi} W_{0}\left[-\frac{1}{k} \exp \left(\frac{\log [(1-x) k !]}{k}\right)\right]}
$$

Proposition IV.3. The Outage probability for Slotted-ALOHA MAC protocol is given by the following expression:

$$
O p^{S A}=\lim _{N \rightarrow+\infty} \frac{1}{N} \sum_{j=1}^{N} \mathbb{I}\left\langle\Sigma_{\alpha}^{j}>\frac{\xi}{\left(\lambda^{*} \pi\right)^{\alpha / 2}}\right\rangle
$$

where $\mathbb{I}\langle$.$\rangle is the indicator function, \lambda^{*}$ defined in (2) and

$$
\begin{aligned}
& \Sigma_{\alpha}^{j}=\log \left(\frac{1}{1-U_{0}^{j}}\right)^{-\alpha / 2} \\
& +\sum_{k=1}^{+\infty}\left(-k W_{0}\left[-\frac{1}{k} \exp \left(\frac{\log \left[\left(1-U_{k}^{j}\right) k !\right]}{k}\right)\right]\right)^{-\alpha / 2} .
\end{aligned}
$$

Proof. The expression (6) gives:

$$
O p=\lim _{N \rightarrow+\infty} \frac{1}{N} \sum_{j=1}^{N} \mathbb{I}\left\langle\left\|X_{0}^{j}\right\|^{-\alpha}+\sum_{k=1}^{+\infty}\left\|X_{k}^{j}\right\|^{-\alpha}>\xi\right\rangle
$$

According to Lemma IV.2 we can express the r.v. $\left\|X_{k}^{j}\right\|$ in terms of a uniform distributed r.v. $U_{k}^{j}$. So, we have:

$$
\begin{aligned}
O p= & \lim _{N \rightarrow+\infty} \frac{1}{N} \sum_{j=1}^{N} \mathbb{I}\left\langle\left(\sqrt{\frac{1}{\lambda \pi} \log \left(\frac{1}{1-U_{0}^{j}}\right)}\right)^{-\alpha}+\right. \\
& \left.\sum_{k=1}^{+\infty}\left(\sqrt{\frac{-k}{\lambda \pi} W_{0}\left[-\frac{1}{k} \exp \left(\frac{\log \left[\left(1-U_{k}\right) k !\right]}{k}\right)\right]}\right)^{-\alpha}>\xi\right\rangle \\
= & \lim _{N \rightarrow+\infty} \frac{1}{N} \sum_{j=1}^{N} \mathbb{I}\left\langle\log \left(\frac{1}{1-U_{0}^{j}}\right)^{-\alpha / 2}+\right. \\
& \left.\sum_{k=1}^{+\infty}\left(-k W_{0}\left[-\frac{1}{k} \exp \left(\frac{\log \left[\left(1-U_{k}^{j}\right) k !\right]}{k}\right)\right]\right)^{-\alpha / 2}>\frac{\xi}{(\lambda \pi)^{\alpha / 2}}\right\rangle
\end{aligned}
$$


And the result holds by setting $\lambda=\lambda^{*}$ by Assumption 3 .

In practice, to compute $O p$, a number of realizations of the random variable $\Sigma_{\alpha}$ are simulated numerically and by counting the number of times that these realizations are higher than the corresponding threshold $\frac{\xi}{\left(\lambda^{*} \pi\right)^{\alpha / 2}}$, one gets to the value of $O p$. Note that for a given value of $\alpha$, we need to estimate the density distribution of $\Sigma_{\alpha}$ only once, indeed, $\Sigma_{\alpha}$ only depends on $\alpha$. Note that our method of computing $O p$ gives similar estimations (that we do not report because of the lack of space) as existing formulas in the literature, but is quite simple in the sens that it involves simulating uniform random variables.

\section{Slotted-ALOHA With CHANNEL RESERVATION AND WITHOUT INTERFERENCE}

To evaluate the impact of interference, we consider in this section a Slotted-ALOHA protocol with a preliminary step of channel reservation (SACR), described in the following. We also assume that there are no interfering nodes in the out range of the BTS as illustrated in Fig. 2. We will see that if all nodes under the range of the BTS are following the SACR strategy, there will be at most only one communicating node by time slot and by frequency channel as illustrated in Fig. 2 .

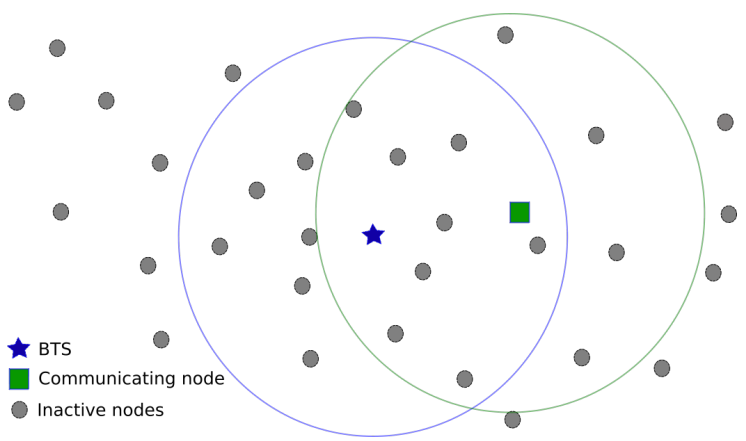

Fig. 2. Illustration of Slotted-ALOHA with channel reservation assuming no interfering nodes in the out range of the BTS.

\section{A. Slotted-ALOHA protocol description}

We consider a Slotted-ALOHA protocol with a preliminary step of reservation. Such as illustrated in Fig. 3, the considered node is initially in "Sleep" state, which corresponds to the state where its radio is switched off. When a packet has to be sent, a Time Slot (TS) and a Frequency Channel (FC) are randomly chosen. The considered node listens to the selected FC during the chosen TS. Further to it, there are two scenarios, either the selected FC is free at TS or not. In the former case, the node sends its packets and waits for an ACK. In the latter case, the considered node repeats this process in the next TS by keeping the same FC. Note that once the FC is free during a certain TS, these two parameters are saved by the node for next transmissions. One of the key performance characteristics of the proposed protocol is the probability of success in transmitting a packet after a limited number of attempts for a new node attempting to join the

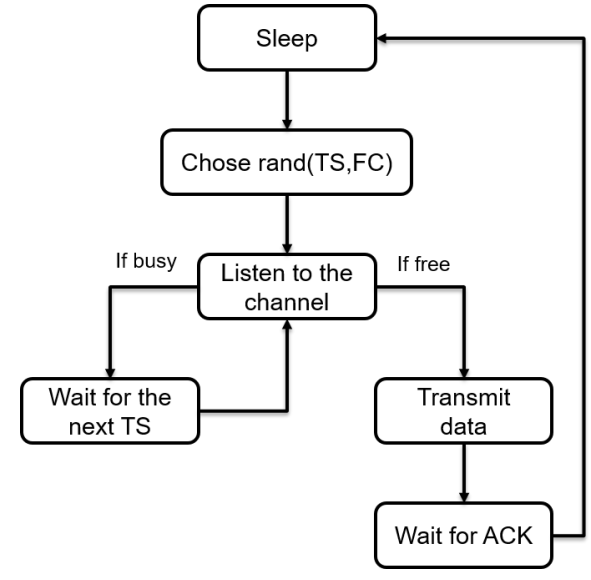

Fig. 3. The channel reservation process followed by a node attempting to join the network.

network. To estimate it, the behavior of the considered node is described by a Markov chain, presented in Fig 4. As shown in this figure, the Markov chain contains $4 n+2$ states, where $n$ is the number of allowed trials to the considered node to join the network (i.e. send its first packet successfully). This latter starts in the "sleep" state and can hop into the "reserve success" or "reserve failed" states with probabilities $R p$ and $1-R p$ respectively. After a successful reservation, the node can transmit its data with probability $(1-O p)^{2}$, or the transmission will be interrupted with probability $O p(2-O p)$ where $O p$ corresponds to the outage probability calculated in the previous section and is equal to 0 when there is no interfering nodes, and $R p$ is the reservation probability which is developed in the next subsection.

\section{B. Reservation probability}

The MAC protocol strategy that we consider is based on Slotted-ALOHA with a channel reservation part, the idea is that each node chooses randomly one FC and one TS before transmitting its first packet and these two parameters (FC,TS) are saved for next transmissions. Let $N_{f c}, N_{t s}$ and $N_{n}$ be respectively the number of available frequency channels, the number of available time-slots and the number of nodes in the network. Using the protocol described above, the number of nodes that have chosen a couple (FC,TS) will be in average $\mu=\frac{N_{n}}{N_{t s} N_{f c}}$. Let $N$ be the random variable representing the number of nodes by couple (FC,TS), we assume that $N$ follows a Poisson law of parameter $\mu$ ie $N \sim \operatorname{Poiss}(\mu)$. The reservation probability $R p$ can be defined as:

$$
R p=\mathbb{P}(N=0)=e^{-\frac{N_{n}}{N_{t s} N_{f c}}}
$$

\section{Transmission success likelihood}

In this subsection, we define the transmission success likelihood metric, noted $T x_{i}$, where $i$ is the number of trials before a successful transmission, to determine the ability for a given node to transmit its packets successfully. Let $\Gamma$ be the transition matrix of the Markov chain described above, the distribution 


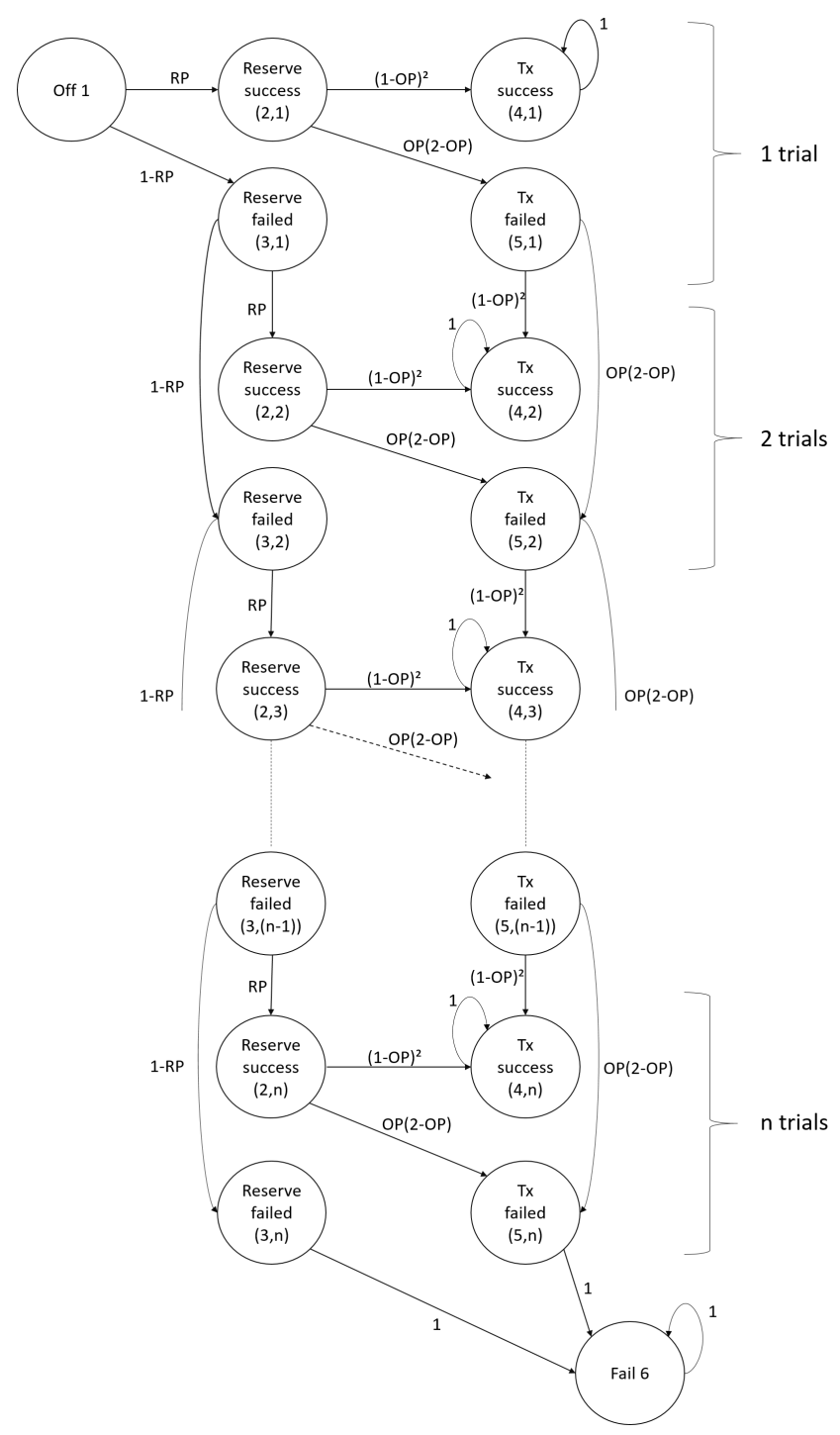

Fig. 4. Markov chain graph modeling the different states of a given node in a distributed WSN, considering a Slotted-ALOHA MAC protocol with channel reservation.

over states can be written as a stochastic row vector $\pi$ such that $\pi^{(\mathrm{k}+1)}=\pi^{(\mathrm{k})} \Gamma$. We have so $\pi^{(\mathrm{k})}=\pi^{(0)} \Gamma^{\mathrm{k}}$. And the infinite distribution is given by the following expression:

$$
\pi^{\infty}=\pi^{0} \lim _{\mathrm{k} \rightarrow \infty} \Gamma^{\mathrm{k}}
$$

Using Equation (12), the transmission success likelihood after $i \in[1, n]$ trial(s) corresponds to the probability that the node is in State $(4, i)$ and it can be calculated as follows:

$$
T x_{i}=\left[\lim _{k \rightarrow \infty} \Gamma^{k}\right]_{(1,4 i)}
$$

where $[M]_{(i, j)}$ denotes the coefficient at line $i$ and column $j$ of the matrix $M$.

\section{SLOTTED-ALOHA With CHANNEL RESERVATION AND INTERFERENCE}

In practice a deployment of a WSN requires several base stations to cover a big area. In such a case, there will be interfering nodes in the out range of a given BTS. In this section, we assume being in this case and we study how these interfering nodes impact the performances of the previous protocol (Slotted-ALOHA with channel reservation). Indeed, as illustrated in Fig. 5, the red points represent interfering nodes that are in the out range of the BTS. Let $R$ be the radius of the circle representing the range of the BTS. To take account of these interfering nodes, we need to calculate the outage probability conditionally to the fact that the first interferer $X_{0} \in \Pi_{\lambda^{*}}$ is out range of the BTS. Formally:

$$
O p^{\left\|X_{0}\right\|>R}=\mathbb{P}\left(\Sigma_{\alpha}>\frac{\xi}{\left(\lambda^{*} \pi\right)^{\alpha / 2}} \mid\left\|X_{0}\right\|>R\right)
$$

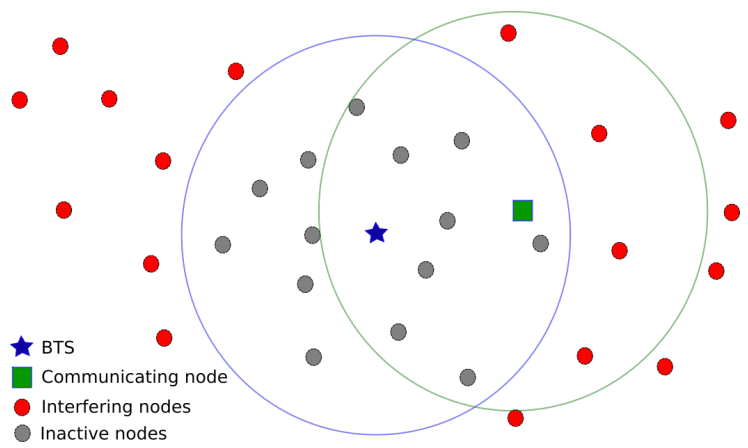

Fig. 5. Slotted-ALOHA with channel reservation and presence of interfering nodes in the out range of the BTS

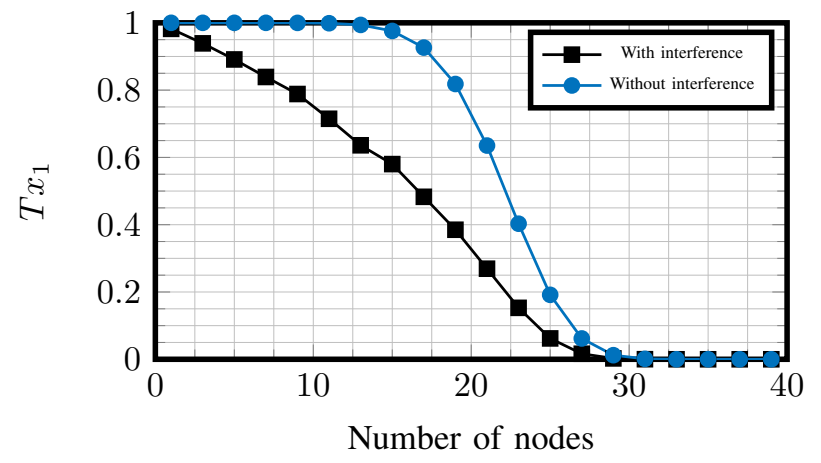

Fig. 6. Comparison of the transmission success likelihood after one trial with no interfering nodes (in blue) and with interference (in black).

As shown in Fig. 6, the transmission success likelihood is influenced by the presence of interfering nodes in the out range of the BTS. Indeed, in this figure we have compared the successful transmission likelihood after one trial with no interfering nodes (in blue) and with interference (in black). We notice thus that the performance of the network is influenced by the interference. 


\section{EXPERIMENTAL ANALYSIS AND MAIN RESULT}

The main objective of this section is to validate experimentally the theoretical expression of the transmission success likelihood obtained in the previous section and to present the main result brought by this paper. In order to corroborate the theoretical transmission success likelihood $T x_{i}$, we estimate its value experimentally by implementing the SACR strategy in real nodes using the Fit-IoT-Lab platform [8]. Indeed, we consider the following experiment: we consider a network composed by 40 nodes that share 20 channels, every node in the network tries to reserve a channel a number of times and sends its packets on the chosen channel if free, by counting the number of successful transmissions we can manage to estimate the values of $T x_{i}$. Note that for simplicity we only show the results for $T x_{1}$ i.e. the transmission success likelihood after one attempt. Indeed, Fig. 7 shows the comparison between the theoretical transmission success likelihood (blue curve) and its experimental estimations (gray curves) for $T x_{1}$. The experiment has been realized five times for consistency. We notice from this figure that the mean over the number of experiments (red curve) is close to the expected theoretical result which validates our theoretical result.

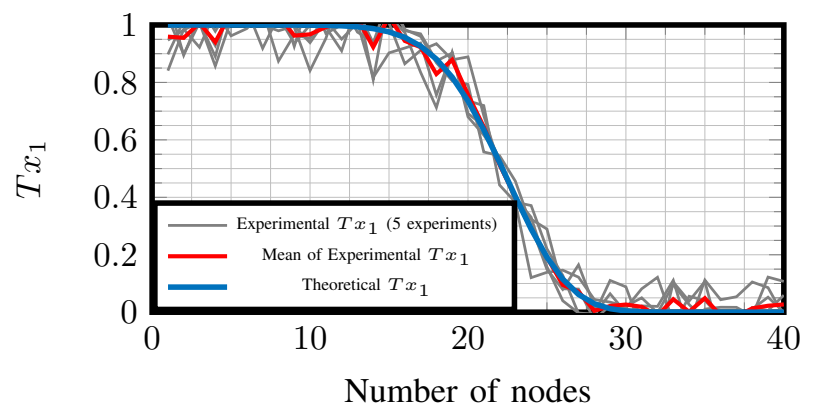

Fig. 7. Comparison between the theoretical transmission likelihood and its practical estimation for 20 channels. Best view in color.

The main result brought by this paper consists in determining for a given wireless sensor network (fixed number of nodes), the necessary number of channels that assures a high transmission success likelihood after a certain number of trials, knowing that a channel is determined by a frequency channel and a time slot. Indeed, as shown in Fig. 8 for a given number of nodes and a desirable transmission success likelihood, our model gives precisely the number of necessary channels, for instance, for $N_{n}=60$ nodes and a transmission success likelihood after one attempt of $T x_{1}=0.97$, the necessary number of channels is $N_{T S} N_{F C}=80$.

\section{CONCLUSION}

To conclude, we have developed in this paper an explicit formula of outage probability in the case of Slotted-ALOHA MAC protocol to highlight the influence of interference. We also studied the Slotted-ALOHA MAC protocol with channel reservation by modeling a given node as a Markov chain. This latter, aims us to define a metric, which is the transmission success likelihood. With this metric, we studied the performance of Slotted-ALOHA with channel reservation strategy in the

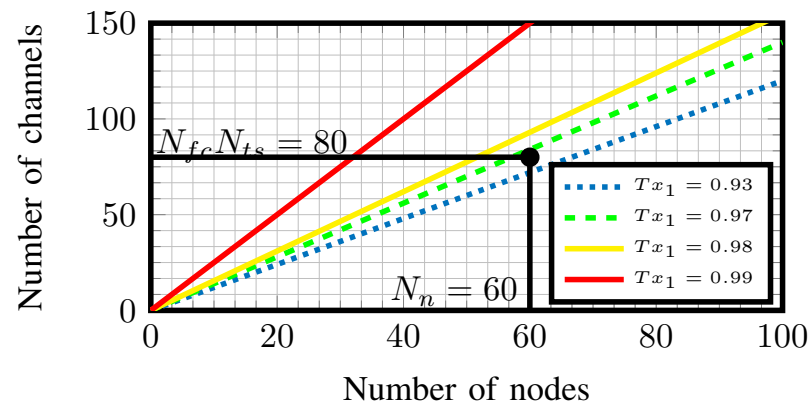

Fig. 8. Transmission success likelihood in terms of number of nodes in the network and number of channels assuming the presence of interfering nodes in the out range of the BTS.

case of no interfering nodes and the presence of interfering nodes. At the end, using this model, we defined a criteria to chose the number of necessary channels for a given network.

\section{ACKNOWLEDGMENTS}

The authors would like to acknowledge support from CREP DATA and LIRIMA PREDNET.

\section{REFERENCES}

[1] S. S. Iyengar and R. R. Brooks, Distributed sensor networks: sensor networking and applications. CRC press, 2016.

[2] N. A. Pantazis, S. A. Nikolidakis, and D. D. Vergados, "Energyefficient routing protocols in wireless sensor networks: A survey," IEEE Communications surveys \& tutorials, vol. 15, no. 2, pp. 551-591, 2013.

[3] N. Ahmed, S. K. Salil, and S. Jha, "Mitigating the effect of interference in wireless sensor networks," in Local Computer Networks (LCN), 2010 IEEE 35th Conference on, Oct 2010, pp. 160-167.

[4] P. Huang, L. Xiao, S. Soltani, M. W. Mutka, and N. Xi, "The evolution of mac protocols in wireless sensor networks: A survey," IEEE communications surveys \& tutorials, vol. 15, no. 1, pp. 101-120, 2013.

[5] V. Toldov, R. Igual-Pérez, R. Vyas, A. Boé, L. Clavier, and N. Mitton, "Experimental Evaluation of Interference Impact on the Energy Consumption in Wireless Sensor Networks," in WoWMoM, Coimbra, Portugal, Jun. 2016

[6] P. M. Pawar, R. H. Nielsen, N. R. Prasad, and R. Prasad, "Mobility impact on cluster based mac layer protocols in wireless sensor networks," Wireless personal communications, vol. 74, no. 4, pp. 1213-1229, 2014.

[7] I. W. Renner and D. I. Warton, "Equivalence of maxent and poisson point process models for species distribution modeling in ecology," Biometrics, vol. 69, no. 1, pp. 274-281, 2013.

[8] E. Fleury, N. Mitton, T. Noel, and C. Adjih, "Fit iot-lab: The largest iot open experimental testbed," ERCIM News, no. 101, p. 4, 2015.

[9] S. Weber, J. G. Andrews, and N. Jindal, "An overview of the transmission capacity of wireless networks," IEEE Transactions on Communications, vol. 58, no. 12, pp. 3593-3604, 2010.

[10] S. Weber, J. G. Andrews et al., "Transmission capacity of wireless

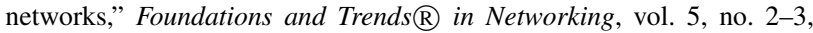
pp. 109-281, 2012.

[11] Z. Gong and M. Haenggi, "Interference and outage in mobile random networks: Expectation, distribution, and correlation," IEEE Transactions on Mobile Computing, vol. 13, no. 2, pp. 337-349, 2014.

[12] H. ElSawy, E. Hossain, and M. Haenggi, "Stochastic geometry for modeling, analysis, and design of multi-tier and cognitive cellular wireless networks: A survey," IEEE Communications Surveys \& Tutorials, vol. 15, no. 3, pp. 996-1019, 2013.

[13] V. R. Gannapathy, A. F. B. T. Ibrahim, Z. B. Zakaria, and M. K. B. Suaidi, "Medium access control (mac) protocol in wireless network: A review."

[14] S. Weber, X. Yang, J. Andrews, and G. de Veciana, "Transmission capacity of wireless ad hoc networks with outage constraints," IEEE Transactions on Information Theory, vol. 51, no. 12, pp. 4091-4102, 12 2005 . 